\title{
Editorial: In Honour of Paul Erdős
}

On 26th March 1993 Paul Erdös turned 80. To celebrate this happy occasion, mathematicians from all over the world gathered in Trinity College, Cambridge for four days of lectures, discussions and a formal banquet on the eve of the birthday.

As all mathematicians know, Paul Erdős has had a profound influence on mathematics in this century: he has made outstanding contributions to number theory, set theory, geometry, complex function theory, probability theory, algebra and interpolation theory. Above all, Paul Erdös is, more than anyone else, the founder of modern combinatorics, especially extremal and probabilistic combinatorics.

Combinatorics, Probability and Computing is therefore especially delighted to dedicate the next four issues to Professor Paul Erdős in his 80th year. During this special 'Erdös year', alongside articles submitted in the regular fashion, the journal will publish papers from the Cambridge Combinatorial Conference in honour of Paul Erdoss.

Each paper carries its own dedication; it is a measure of the wide-ranging nature of Professor Erdös' influence on mathematics that such a wide variety of papers can be published in his honour.

May he continue to enrich us with the originality of his insight and imagination, as well as inspiring us with myriads of exciting problems well into the next millennium.

Béla Bollobás 